\title{
The Role of NOMs in Stabilizing and Destabilizing Clay Particles in Aquatic Environment
}

\author{
Qianyue Guo ${ }^{1, a}$, Jin Guo ${ }^{2, b}$ and Ni Liang ${ }^{\star^{1, c}}$ \\ ${ }^{1}$ Faculty of Environmental Science and Engineering, Kunming University of Science \& Technology, \\ Kuming 650500, China; \\ ${ }^{2}$ Honghe Environmental Protection Agency, Mengzi 661199, China. \\ a404726372@qq.com, b327054652@qq.com, c751047048@qq.com
}

Keywords: Clay minerals, NOMs, Environmental process, Aggregation.

Abstract. Clay minerals are an important constituent of soils and it could disperse in aquatic environment which indicates they would have interaction with natural organic matters (NOMs). Although there is some researchers work on the aggregation kinetic process of clay particles in the presence of NOMs [1, 2], few study is focus on the change caused by varying concentration of NOMs. This study examined the hydrodynamic diameters of Na-kaolnite particles under varying concentration of TA and electrolytes. The existing of high concentration NOMs could destabilize the dispersed Na-kaolnite particles in aquatic environment while the low concentrations display in an opposite way.

\section{Introduction}

Clay minerals are an important constituent of soils and it could disperse in aquatic environment because of the terminal Al-OH or Si-OH sites. According to some previous research, the clays show a permanent negative charge within the structure at the same time as a variable charge at the particle edges [1, 3]. Some researchers have proven that clay particles can destabilize both positively and negatively charged ENPs under various conditions in the subsurface and open water bodies $[4,5]$. Presently, majority of the studies illustrate natural organic matter (NOM) mediated fate and transport of ENPs. The structure of particle network in soils, the retardation or release of colloidal particles, and their mobility and transport are inherently influenced by natural organic matter bound to the mineral matrix [6]. There is some paper focus on the hetero-aggregation of clay particles with NOMs [1, 2], but just few about the effects of concentration of NOMs.

As newly formed landscapes evolve, physical and biological changes occur that are collectively known as primary succession include aquatic environments [7]. Although the basic concept is ecological, there is no absolutely independent process in Environment. In this study, we tried to prove that the NOMs are of importance in the bridging of clay particles in aquatic environment so that they may have influence in the primary succession in aquatic environments.

\section{Materials and Methods}

Preparation of Na-Kaolinite. Portions of the kaolinite $(10 \mathrm{~g})$ were mixed with $250 \mathrm{~mL}$ Milli-Q water and sonicated for one hour to get a kaolinite suspension. The fraction $<1 \mu \mathrm{m}$ was then separated by several centrifugation $(3000 \mathrm{r} / \mathrm{min}$ for $20 \mathrm{mins})$ and redispersion steps. The $<1 \mu \mathrm{m}$ fraction was collected and washed five times with $1 \mathrm{M} \mathrm{NaCl}$ solution to saturate the clay with $\mathrm{Na}^{+}$. The Na saturated kaolinite was then washed free of excess salt with Milli-Q water and freeze dried.

Preparation of TA solutions. Tannic acid was purchased from Sigma Chemical. Tannic acid has been used in previous research as a reproducible surrogate for natural organic matters (NOMs). The molecular mass appears to approximate mean values reported for surface waters. Solutions of TA were made at $1 \mathrm{~g} / \mathrm{L}$ then adjusted its $\mathrm{pH}$ to 7 and stored refrigerated in sterile tubes to avoid potential microbial interference.

Zeta potential measurements. THE electrophoretic mobility and hydrodynamic diameter of particles were measured by a Zeta PALs (Brookhaven, America) equipped with a folded capillary 
cell at $25^{\circ} \mathrm{C}$. Predetermined amounts of $500 \mathrm{mM} \mathrm{CaCl}_{2}$ and $1 \mathrm{M} \mathrm{NaCl}$ solution were added to the kaolinite suspension to get desired ionic strengths. The $\zeta$ of pure TA were measured at a concentration of $1 \mathrm{~g} / \mathrm{L}$ while that of Na-kaolinite is just $10 \mathrm{mg} / \mathrm{L}$. We had to use the high concentration of $1 \mathrm{~g} / \mathrm{L}$ for TA to get enough intensity of the back-scattered light.

Aggregation kinetics studies. Growth rate of various particles aggregates were also measured using the Zeta PALs (Brookhaven). All the experiments were run in triplicate or more. Collected data were analyzed and plotted against the measurement time. At a fixed angle scattering experiment setup the initial rate of fullerene particles aggregation can be express as:

$$
\left[\frac{d_{\alpha_{h}(t)}}{d_{t}}\right]_{t \rightarrow 0} \propto k_{11} N_{0}
$$

where $\alpha_{h}(t)$ is the hydrodynamic radius of Na-kaolinite particles at time $t, \mathrm{~N}_{0}$ the initial number of particles, and $\mathrm{k}_{11}$ the initial aggregation rate constant. The left side of this equation is the slope of Na-kaolinite aggregate size vs. time which can be estimated using the collected DLS data. Increasing $\mathrm{Na}^{+}$concentration increased aggregation rate (reaction controlled mecha nism, slow) until a limit, where increasing $\mathrm{Na}^{+}$concentration di not further affect the rate of aggregation (diffusion controlled mechanism, fast). The ratio of $\mathrm{k}_{11}$ in any desired calcium concentration to $\mathrm{k}_{11}$ in the diffusion controlled regime is defined as attachment efficiency $(\alpha)$ or inverse stability ratio $(1 / W)$. Attachment efficiency $(\alpha)$ of Na-kaolnite at different sodium concentrations and constant $N_{0}$ were calculated to quantify kinetics of Na-kaolnite aggregation using the following equation:

$$
\alpha=\frac{1}{W}=\frac{k_{11}}{\left(k_{11}\right)_{\text {fast }}}=\frac{\frac{1}{N_{0}}\left(\frac{d_{\alpha_{h}(t)}}{d_{t}}\right)_{t \rightarrow 0}}{\frac{1}{\left(N_{0}\right)_{\text {fast }}}\left(\frac{d_{\alpha_{h}(t)}}{d_{t}}\right)_{t \rightarrow 0 \text {. fast }}}=\frac{\left(\frac{d_{\alpha_{h}(t)}}{d_{t}}\right)_{t \rightarrow 0}}{\left(\frac{d_{\alpha_{h}}}{d_{t}}\right)_{t \rightarrow 0 \text {. fast }}}
$$

\section{Results and discussion}

Characteristics of suspensions. Na-kaolinite suspension (Na-KL) had a transparent white color and the concentration of Na-kaolinite in water was $60 \mathrm{mg} / \mathrm{L}$ which dilute to $15 \mathrm{mg} / \mathrm{L}$ when conduct the experiments. For pure TA, the $\zeta$ at pH7 $(-33.23 \pm 2.12)$ was lower than that at $\mathrm{pH} 4(-15.71 \pm 3.28)$ due to the protonation. The $|\zeta|$ of Na-kaolinite suspension with and without TA at different $\mathrm{pH}$ condition were displayed in Figure 1a. As High $|\zeta|$ of particles in water, as reported in several previous studies, was the reason for high stability of suspension [8]. The fact that TA (1-5 ppm) increased $|\zeta|$ of kaolinite suspension even though their $|\zeta|$ were similar with that of pure Na-kaolinite suspension indicates that the interaction between them was not only electrostatic. However, the $|\zeta|$ decreased as the concentration of TA up to $10 \mathrm{ppm}$, which related to the change of hydrodynamic diameter of particles (Fig.1b). The phenomenon suggested that TA destabilize the clay particles at high concentration.

(a)

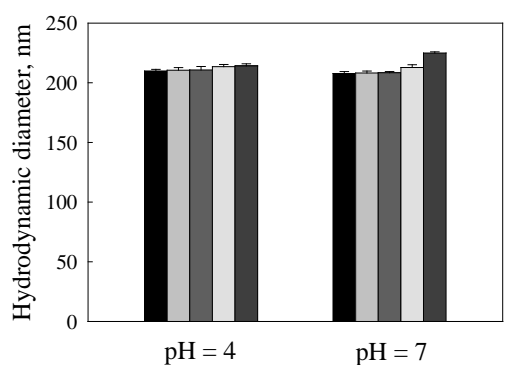

(b)

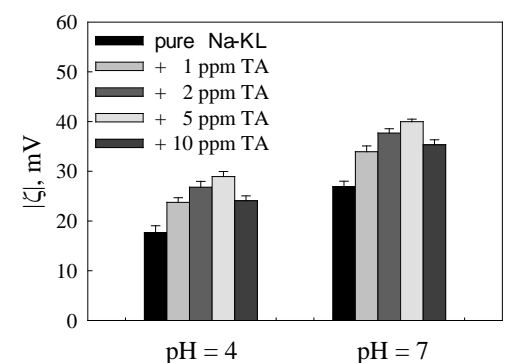

Fig.1 (a) Hydrodynamic diameter of pure Na-KL and addition of TA. (b) The $|\zeta|$ of Na-KL with TA. Addition of TA increased the $|\zeta|$ of Na-kaolinite suspension. The fact that TA increased $|\zeta|$ of kaolinite suspension even though their $|\zeta|$ were similar with that of pure Na-kaolinite suspension 
indicates that the interaction between them was not only electrostatic. Our previous research pointed out that TA increased the $|\zeta|$ of fullerene aggregates as much as other types of NOM, indicating deprotonation of most phenolic groups of TA at pH7 [8].

Homoaggregation of Na-kaolnite. Increasing concentrations of cations were used to initiate particle aggregation. Sodium ions effectively increased the aggregate size immediately after their addition to the stable kaolinite suspension. In Fig.2 two distinct aggregation regimes can be observed: reaction controlled regime, where $\alpha$ increased linearly with increasing $\mathrm{Na}^{+}$, and diffusion controlled regime, where rate of aggregation was controlled by diffusion rate of Na-kaolinite aggregates in the solution. The intercept between two regimes is CCC which was determined equal to $63.67 \mathrm{mM}$ and $244.14 \mathrm{mM} \mathrm{NaCl}$ for Na-kaolinite at $\mathrm{pH} 4$ and $\mathrm{pH} 7$ respectively. The majority of evidence suggests that kaolinite has $\mathrm{pH}$-dependent surface charge on the edges and permanent, negative surface charge on the basal faces of the kaolinite platelets $[1,4,9]$. At $\mathrm{pH} 4$, the edges are positively charged resulting in edge-to-face hetero-coagulation by electrostatic and van der waals attractively forces between positively charged edges and negatively charged faces.

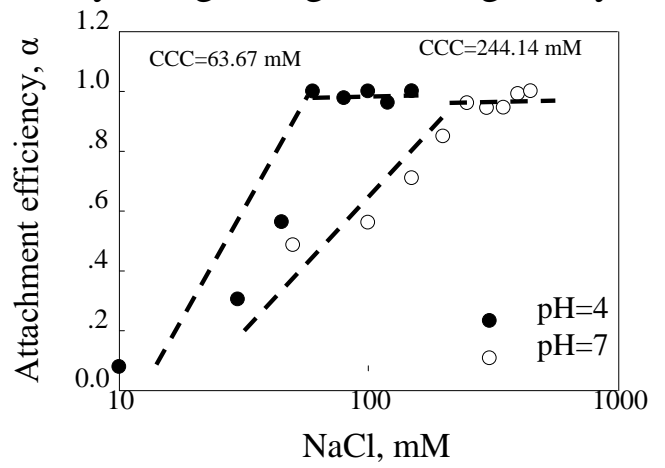

Fig.2 Attachment efficiencies $(\alpha)$ of Na-kaolinite as a function of $\mathrm{Na}^{+}$concentration.

Influence of TA. Tannin is a biodegradable anionic polymer and as a coagulant aid can be a potential substitute for synthetic anionic polyelectrolytes in water treatment [10]. But in our study, the usage of TA as a coagulant aid may be challenged. The aggregation profile showed in Fig.3 gave a unified scene which is similar to the trend of $|\zeta|$. After the addition of divalent calcium ions and univalent sodium ions the particle size increased as time in all system. Small additions of TA caused pronounced increases in colloidal stability because points of Na-KL $+1 \mathrm{ppm}$ TA and Na-KL +2 ppm TA were below pure Na-KL. Increasing ionic strength generally resulted in decreased colloidal stability, indicating that the suspensions were stabilized by electrostatic repulsive forces. Under acidic condition, adsorption of TA to the edges of kaolinite particles can cause charge reversal from positive to negative edge surface charge (Fig1) and enhance the electrostatic repulsion. The sorption of TA to kaolinite may result in increased colloidal stability due to reversal of edge charge and an increase in negative surface charge (electrostatic stabilization). In addition, adsorbed TA may also provide adsorbed polymer chains reaching into the bulk solution. This may result in steric stabilization caused by a thermodynamic repulsion that occurs between interpenetrating organic polymer chains attached to the colloid surfaces [1,11].

(a)

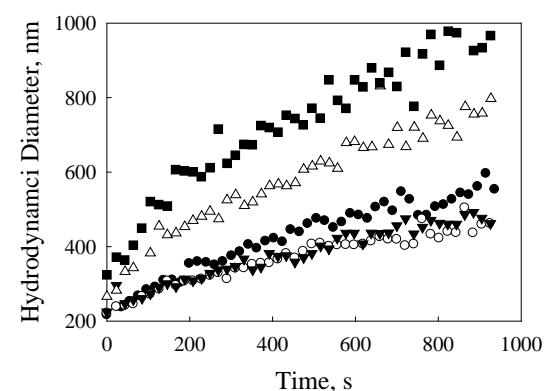

(c) (b)

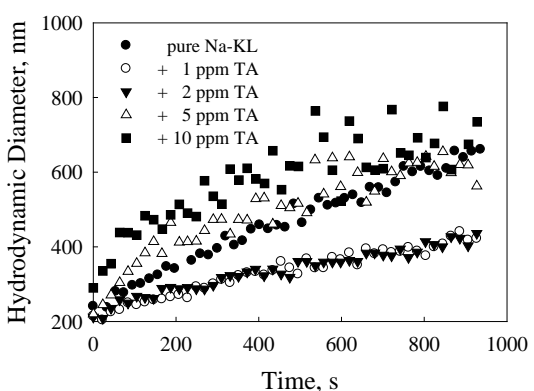

(d) 

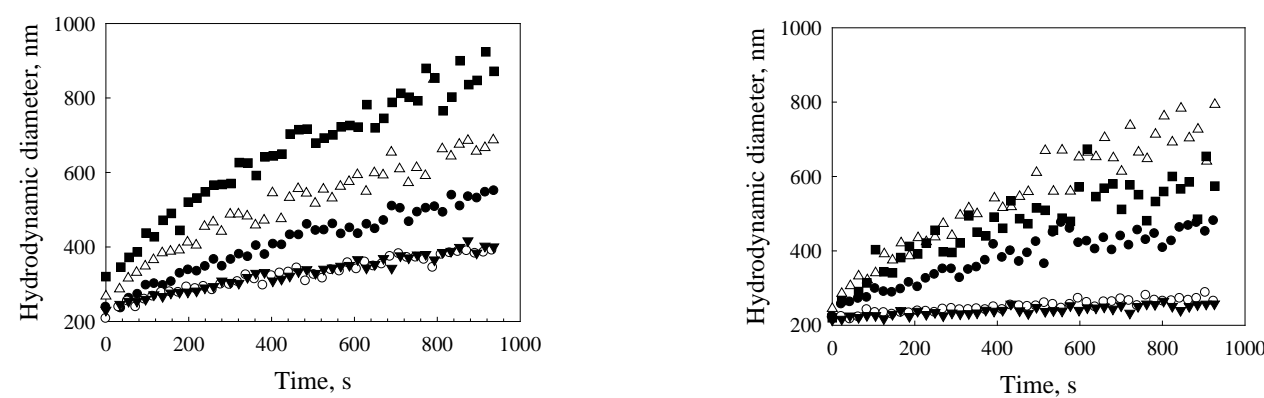

Fig.3 Aggregation profiles of Na-kaolinite (Na-KL) particle size with different amount TA in $5 \mathrm{mM}$ $\mathrm{Ca}^{2+}$ at pH4 (a) and pH7 (b), in $50 \mathrm{mM} \mathrm{Na}^{+}$at pH4 (c) and in $150 \mathrm{mM} \mathrm{Na}^{+} \mathrm{pH} 7$ (d).

Nevertheless, the scene reversed as the TA increased. The points of Na-KL $+5 \mathrm{ppm}$ TA and Na-KL $+10 \mathrm{ppm}$ TA were over pure Na-KL. The mechanism was similar to low concentration of TA initially. Adsorbed TA may also provide adsorbed polymer chains reaching into the bulk solution. The organic polymer chains attached to the colloid surfaces may approach to each other if the concentration of them were high enough due to the aromatic ring in TA. The $\pi-\pi$ interaction could be the mechanism.

The eutrophication resulting from excessive inputs of nutrients and toxic substances, species invasions and extirpations happened in the primary succession of an aquatic area. These process allowed accumulation of organic matter result in destabilizing suspended clay particles, in turn facilitate the primary succession. Then the lake may change into a wetland or even an island.

\section{Summary}

TA significantly influences the association of kaolinite particles dispersed in water. The enhanced colloidal stability at low concentration of TA is due to the reversal of edge charge and an increase in negative surface charge (electrostatic stabilization) and steric stabilization caused by a thermodynamic repulsion that occurs between interpenetrating organic polymer chains attached to the colloid surfaces. However, the $\pi-\pi$ interaction between TA molecular could force the dispersed clay particles approach to each other once the concentration reached a high level. These mechanisms could help us to understand the role of NOMs in the subsurface water and surface water especially in a primary succession of aquatic succession. The existing of high concentration NOMs could facilitate the succession while the low concentration may hander it.

\section{Acknowledgements}

This research was supported by National Scientific Foundation of China (41573100).

\section{References}

[1] Kretzschmar, R., H. Holthoff and H. Sticher, Influence of $\mathrm{pH}$ and humic acid on coagulation kinetics of kaolinite: A dynamic light scattering study. Journal of Colloid and Interface Science, 1998. 202(1): p. 95-103.

[2] Zhang, H.C., S. Taujale, J.Z. Huang, et al., Effects of NOM on Oxidative Reactivity of Manganese Dioxide in Binary Oxide Mixtures with Goethite or Hematite. Langmuir, 2015. 31(9): p. 2790-2799.

[3] Tertre, E., S. Castet, G. Berger, et al., Surface chemistry of kaolinite and Na-montmorillonite in aqueous electrolyte solutions at 25 and 60 degrees C: Experimental and modeling study. Geochimica Et Cosmochimica Acta, 2006. 70(18): p. 4579-4599.

[4] Wang, H.T., Y.N. Dong, M. Zhu, et al., Heteroaggregation of engineered nanoparticles and kaolin clays in aqueous environments. Water Research, 2015. 80: p. 130-138. 
[5] Zhou, D.X., A.I. Abdel-Fattah and A.A. Keller, Clay Particles Destabilize Engineered Nanoparticles in Aqueous Environments. Environmental Science \& Technology, 2012. 46(14): p. 7520-7526.

[6] Etelka Tombacz, Z.L., Erzsebet Illes, Andrea Majzik, Erwin Klump, The role of reactive surface sites and complexation by humic acids in the interaction of clay mineral and iron oxide particles. Organic Geochemistry, 2004. 35: p. 257-267.

[7] Engstrom, D.R., S.C. Fritz, J.E. Almendinger, et al., Chemical and biological trends during lake evolution in recently deglaciated terrain. Nature, 2000. 408(6809): p. 161-166.

8. Mashayekhi, H., S. Ghosh, P. Du, et al., Effect of natural organic matter on aggregation behavior of C-60 fullerene in water. Journal of Colloid and Interface Science, 2012. 374: p. 111-117.

[9] Tombacz, E. and M. Szekeres, Surface charge heterogeneity of kaolinite in aqueous suspension in comparison with montmorillonite. Applied Clay Science, 2006. 34(1-4): p. 105-124.

[10]Ozacar, M. and I.A. Sengil, Evaluation of tannin biopolymer as a coagulant aid for coagulation of colloidal particles. Colloids and Surfaces a-Physicochemical and Engineering Aspects, 2003. 229(1-3): p. 85-96.

[11] Li, Q., B. Xie, Y.S. Hwang, et al., Kinetics of C-60 Fullerene Dispersion in Water Enhanced by Natural Organic Matter and Sunlight. Environmental Science \& Technology, 2009. 43(10): p. 3574-3579. 